\title{
Stochastic Model Predictive Control Approaches applied to Drinking Water Networks
}

\author{
Juan M. Grosso ${ }^{1}$, Pablo Velarde ${ }^{2}$, Carlos Ocampo-Martinez ${ }^{1}$, José M. Maestre ${ }^{2}$ \\ and Vicenç Puig ${ }^{1}$ \\ ${ }^{1}$ Automatic Control Department, Universitat Politècnica de Catalunya (UPC), \\ Institut de Robòtica i Informàtica Industrial (CSIC-UPC), \\ Llorens i Artigas, 4-6, 08028 Barcelona, Spain. \\ ${ }^{2}$ Departamento de Ingeniería de Sistemas y Automática, Universidad de Sevilla, \\ Escuela Superior de Ingenieros, Camino de los Descubrimientos s/n, 41092 Sevilla, Spain.
}

\begin{abstract}
Control of drinking water networks is an arduous task given their size and the presence of uncertainty in water demand. It is necessary to impose different constraints for ensuring a reliable water supply in the most economic and safe ways. To cope with uncertainty in system disturbances due to the stochastic water demand/consumption, and optimize operational costs, this paper proposes three stochastic model predictive control (MPC) approaches, namely: chance-constrained MPC, tree-based MPC, and multiplescenarios MPC. A comparative assessment of these approaches is performed when they are applied to real case studies, specifically, a sector and an aggregate version of the Barcelona drinking water network in Spain.
\end{abstract}

\section{Introduction}

Drinking water networks (DWNs) transport water from sources to consumers ensuring the quality of service [1]. Nevertheless, limited water sources, conservation and sustainability policies, as well as the infrastructure complexity for meeting consumer demands with appropriate flow pressure and quality levels make water management a challenging problem [2]. Water demand forecasting based on historical data is commonly used for the operational control of water supply along a given prediction horizon. However, the optimality of such scheduling is affected by the one associated to water demand forecasts. Therefore, the scheduling of control inputs must be continuously adjusted. This leads to consider the DWNs as dynamical systems and their operation as optimal control problems, with the objective of satisfying water demands in an optimal manner despite the presence of disturbances and uncertainties, and considering additionally issues such as constraints on the manipulated and output variables and multiple conflicting control goals. Given the features of the problem, MPC provides a control framework capable of dealing with these issues in an explicit manner $[2,3]$. The main idea of MPC is to obtain a control signal by solving, at each time step, a finite-horizon optimization problem (FHOP) that takes into account a model of the system to predict its evolution and to steer it in accordance to given objectives. The first component of the obtained control sequence is applied to the DWN at the current time step and the problem is solved again at the next time step, following a receding horizon strategy [4]. There are several examples of MPC applied to water networks in the literature, see e.g., $[5,6,7,8,9]$ and references therein.

Among the aforementioned references, a common approach used to cope with perturbed systems is to rely on the so-called certainty equivalence property [10], which in the MPC framework leads to a perturbed nominal deterministic MPC strategy, also named certainty-equivalent MPC (CE-MPC). This strategy addresses perturbed systems by considering nominal models that do not include the uncertainty. Hence, the expected value of system inputs will lead to an average performing system. In the case of linear systems with uniformly distributed scenarios, the certainty equivalence property holds [11] and this strategy is optimal. 
Nevertheless, this may not be the case due to factors such as the presence of nonlinearities. Hence, the CEMPC is usually complemented with a (de)tuning of the controller. Although, in one hand, a frequent violation of soft constraints can occur, on the other hand, infeasible solutions would result if the constraints were hard due to the ignored effects of future uncertainty. Nevertheless, there exist other MPC schemes reported in the literature that aim to ensure robust stability and compliance with constraints in the presence of stochastic disturbances, see e.g., [12, 13]. As summarized in [13], alternative approaches of MPC for stochastic systems are based on min-max MPC, tube-based MPC, and stochastic MPC (SMPC). The first two approaches are oriented to ensure worst-case robustness and consequently are conservative, while the third approach relies on stochastic programming (SP) techniques to offer a probabilistic constraint fulfilment [14]. Since some violations are allowed, the solutions obtained are less conservative and hence the performance is better in terms of cost from the objective function. In this way, disturbances are modelled as random variables, and the control problem is stated by using the expected value of the system variables, i.e., states and control inputs.

The stochastic approach is a mature theory in the field of optimization [15], but renewed attention has been given to it due to its great potential in control applications, see e.g., [16] and references therein. From the wide range of SMPC methods, this paper focuses on three specific techniques, namely: chance-constrained $M P C$ (CC-MPC), tree-based MPC (TB-MPC), and multiple-scenario MPC (MS-MPC), also called Multiple $\mathrm{MPC}$ in [17].

CC-MPC is a stochastic control strategy that provides robustness in terms of probabilistic (chance) constraints, such that the probability of violation of any operational requirement or physical constraint is below a prescribed value [18]. Some works that address the CC-MPC approach in water systems are $[19,20,21]$ and references therein. On the other hand, in the TB-MPC, uncertainty is addressed by considering simultaneously a set of possible disturbance scenarios modelled as a rooted tree, which branches along the prediction horizon with a common initial value of the disturbances, see, e.g., [22, 23, 24]. This technique computes a set of control input sequences, one per each scenario in the disturbance tree, but only applies the first element from all sequences, which is the same for all the possible scenarios considered. At the next time instant, the control inputs are recalculated and only the value of the control sequence that corresponds to the first time instant is applied. The MS-MPC follows a similar approach, but it computes a single control sequence instead of a tree of control inputs. To do so, the MS-MPC takes into account also different possible evolutions of the process disturbances but not necessarily in the form of a tree [12]. This approach was used in [17] for designing an MPC controller for drainage systems. In this way, both the TB-MPC and the MS-MPC incorporate robustness by considering several disturbance scenarios in a single optimization problem.

The main contribution of this paper consists in the design and assessment of the three aforementioned stochastic controllers, i.e., CC-MPC, TB-MPC, and MS-MPC, applied to the operational management of a real DWN, i.e., the Barcelona DWN. These approaches offer a compromise between economic costs, reliability and computational burden. A discussion of the advantages and weaknesses of these control approaches in the sense of tractability and performance is presented when applied to two case studies, i.e., a small-scale and a large-scale model of the Barcelona DWN. This paper is an extension of the results presented in [21], which details the CC-MPC controller design for DWN, and [25], which provides a comparison between TB-MPC and CC-MPC applied to DWN.

The remainder of the paper is organized as follows. Section 2 describes the case studies based on the Barcelona DWN and discusses the modelling of water demand. Section 3 describes the DWN control problem and introduces the CE-MPC formulation. Section 4 describes the formulation of the CC-MPC, TB-MPC and MS-MPC approaches. Section 5 discusses the results of the application of the three proposed SMPC approaches to the case studies via simulation. Finally, the conclusions are drawn in Section 6.

Notation. Throughout this paper, $\mathbb{R}, \mathbb{R}^{n}, \mathbb{R}^{m \times n}$ and $\mathbb{R}_{+}$denote the field of real numbers, the set of column real vectors of length $n$, the set of $m$ by $n$ real matrices and the set of non-negative real numbers, respectively. Moreover, $\mathbb{S}_{++}^{n}$ denotes the set of positive definite matrices of dimension $n$, while $\mathbb{Z}_{+}$denotes the set of non-negative integer numbers including zero. $\mathbb{Z}_{[a, b]} \triangleq\left\{x \in \mathbb{Z}_{+} \mid c_{1} \leq x \leq c_{2}\right\}$ for some $c_{1}, c_{2} \in \mathbb{Z}_{+}$and $\mathbb{Z}_{\geq c} \triangleq\left\{x \in \mathbb{Z}_{+} \mid x \geq c\right\}$ for some $c \in \mathbb{Z}_{+}$. For a vector $x \in \mathbb{R}^{n}, x_{(i)}$ denotes the $i$-th element of $x$. Similarly, $X_{(i)}$ denotes the $i$-th row of a matrix $X \in \mathbb{R}^{n \times m}$. Additionally, $\|\cdot\|_{Z}$ denotes the weighted 2-norm of a vector, i.e., $\|x\|_{Z}=\left(x^{\top} Z x\right)^{1 / 2}$. If not otherwise indicated, all vectors are column vectors. Transposition is denoted by superscript ${ }^{\top}$, similarity is denoted by $\sim$, and the operators $<, \leq,=,>, \geq$ denote element-wise relations 
of vectors. Furthermore, 0 denotes a zero column vector and $I$ the identity matrix, both of appropriate dimensions.

\section{Case Studies Description and Preliminaries}

The MPC approaches presented in this paper are assessed with two representative case studies based on the Barcelona DWN.

A DWN must satisfy water demands and guarantee service reliability by making optimal use of water sources and network components in order to minimize economic costs. The water network operates as a fullinterconnected system driven by endogenous and exogenous flow demands. In the Barcelona DWN, water is taken from both superficial and underground sources. Flows coming from sources are regulated by pumps or valves. After being extracted from sources, water is purified up to levels suitable for human consumption in four water treatment plants (WTP). The water flow from any of the sources is limited and has costs associated to the extraction and the treatment required. The DWN is divided in two management layers: the transport network, which links the water treatment plants with the reservoirs located all over the city, and the distribution network, which is sectored in sub-networks, linking reservoirs directly to consumers. In this work, each sector of the distribution network is considered as a pooled demand to be satisfied by the transport network.

The two systems under study have been extracted from the Barcelona transport network. The first case study consists in a sector model and the second one is an aggregate model of the whole network. They differ mainly in the size of the network flow problems and the number of constitutive elements:

- The sector network considers only a small-scale subsystem related to a portion of the overall DWN (see Fig. 1). This case study considers 2 water sources, 3 tanks, 6 flows controlled by valves and pumps, 4 demand nodes and 2 intersection nodes.

- The aggregate network represents a simplification from the original DWN, where sets of elements are aggregated in a single element in order to reduce the size of the original model (see Fig. 2). It consists of 9 water sources, 17 tanks, 61 flows controlled by valves and pumps, 25 demand nodes and 11 intersection nodes.

\subsection{Demand Modelling and Scenario Generation}

In DWNs, the uncertainty is generally introduced by the stochastic behaviour of water consumers. Therefore, a proper demand modelling is required to achieve an acceptable water supply service level. For the case studies of this paper, time series forecasting based on auto-regressive integrated moving average (ARIMA) models are used due to its ability to capture complex linear dynamics from historical data [26]. In this way, it is possible to generate artificial scenarios with similar statistical properties to those obtained from historical data.

A correct sampling of scenarios is essential for developing the proposed SMPC approaches. For the CC-MPC approach, ARIMA models are used to generate a large number of possible demand scenarios by Monte Carlo sampling for a given time horizon $N \in \mathbb{Z}_{+}$; the mean demand is then used for the controller design. For the MS-MPC approach, a set of $N_{s} \in \mathbb{Z}_{+}$water demand scenarios is generated and used. Increasing the number of scenarios allows the controller to gain robustness but at the expense of additional computational effort and economic performance losses. MS-MPC is generally over-conservative, because it does not consider the controller capacity to adapt to the new observations of the uncertainty and reformulate its controller structure at each time instant. To cope with this drawback, a representative subset of scenarios might be chosen using scenario reduction algorithms [27, 28]. Moreover, the reduced set of scenarios can be transformed into a rooted tree of possible evolutions of the demand [29] that can be used with the TB-MPC approach, see, e.g., [30, 31]. More specifically, a reduction of the initial number of scenarios into a rooted tree of $N_{r}$ scenarios, obtained by generating an ensemble forecast tree, only reduces the number of scenarios that have similar features with their adjacent scenarios. The disturbances tree remains the dominant scenarios. The rationale behind this approach is that uncertainty spreads with time, i.e., it is possible to predict more 


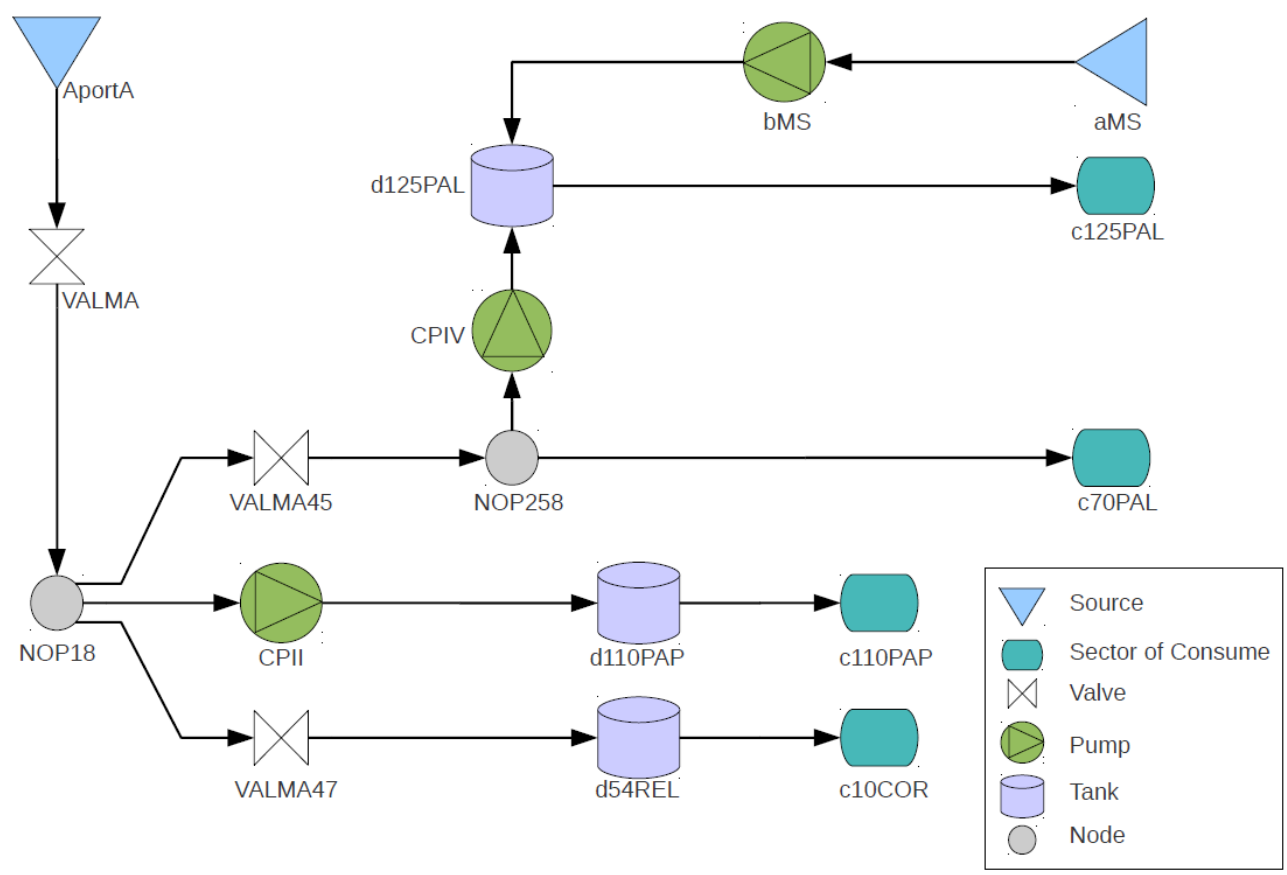

Figure 1: Sector diagram of the Barcelona DWN

accurately the demand evolution in a short time horizon than in a large one. Besides, TB-MPC takes into account, within the optimization problem, the MPC capacity to adapt, i.e., a control input sequence is calculated for each branch of the tree at each time step, by implementing the so-called Multi-stage Stochastic Programming, as pointed in [22].

\section{DWN Control Problem Statement}

This section introduces the CE-MPC formulation, including the system defined by time-invariant state-space linear model in discrete time, its goals and constraints.

\subsection{Control-oriented Model}

The system model may be described considering the volume of water in tanks as the state variables $x \in \mathbb{R}^{n}$, the flow through the actuators as the manipulated inputs $u \in \mathbb{R}^{m}$, and the demanded flows as additive measured disturbances $d \in \mathbb{R}^{p}$. The control-oriented model of the network is described by the following equations for all time instant $k \in \mathbb{Z}_{+}$:

$$
\begin{aligned}
x_{k+1} & =A x_{k}+B u_{k}+B_{p} d_{k}, \\
0 & =E_{u} u_{k}+E_{d} d_{k},
\end{aligned}
$$

where (1a) and (1b) describe the mass balance equations for storage tanks and intersection nodes, respectively. Moreover, $A \in \mathbb{R}^{n \times n}, B \in \mathbb{R}^{n \times m}, B_{p} \in \mathbb{R}^{n \times p}, E_{u} \in \mathbb{R}^{q \times m}$ and $E_{d} \in \mathbb{R}^{q \times p}$, are time-invariant matrices dictated by the network topology.

Assumption 1 The states in $x$ and the demands in $d$ are measured at any time instant $k \in \mathbb{Z}_{+}$.

Assumption 2 The pair $(A, B)$ is controllable and (1b) is reachable ${ }^{1}$, provided that $q \leq m$ with $\operatorname{rank}\left(E_{u}\right)=$ $q$.

\footnotetext{
${ }^{1}$ If $q<m$, then multiple solutions exist, so $u_{k}$ should be selected by means of an optimization problem. Equation (1b) implies the possible existence of uncontrollable flows $d_{k}$ at the junction nodes. Therefore, a subset of the control inputs will be restricted by the domain of some flow demands.
} 


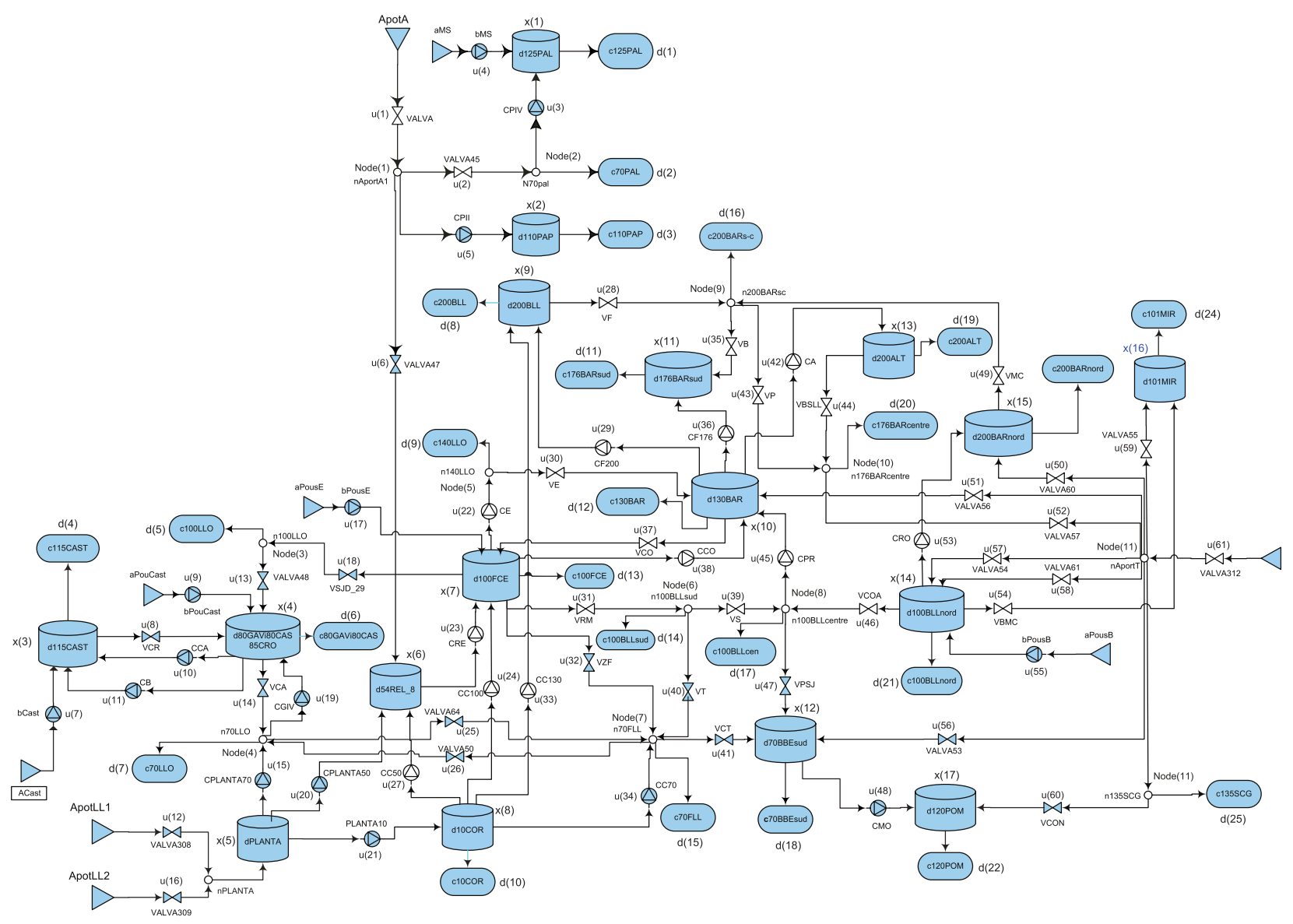

Figure 2: Aggregate model of the Barcelona DWN

Assumption 3 The realization of disturbances at the current time instant $k$ may be decomposed as

$$
d_{k}=\bar{d}_{k}+e_{k}
$$

where $\bar{d}_{k} \in \mathbb{R}^{n_{d}}$ is the vector of expected disturbances, and $e_{k} \in \mathbb{R}^{n_{d}}$ is the vector of forecasting errors with non-stationary uncertainty and a known (or approximated) quasi-concave probability distribution $\mathcal{D}$. Therefore, the stochastic nature of each $j^{\text {th }}$ row of $d_{k}$ is described by $d_{(j), k} \sim \mathcal{D}_{i}\left(\bar{d}_{(j), k}, \Sigma\left(e_{(j), k}\right)\right)$, where $\bar{d}_{(j), k}$ denotes its mean, and $\Sigma\left(e_{(j), k}\right)$ its variance.

Assumption 4 The demands are bounded, i.e., $d_{k} \in \mathbb{D}$, for all $k \in \mathbb{Z}_{+}$, and input-disturbance dominance constraints hold, i.e., $B_{d} \mathbb{D} \subseteq-B \mathbb{U}$ and $E_{d} \mathbb{D} \subseteq-E_{u} \mathbb{U}$.

The system is subject to storage and flow capacity hard constraints considered here in the form of convex polyhedra defined as

$$
\begin{aligned}
& x_{k} \in \mathbb{X} \triangleq\left\{x \in \mathbb{R}^{n} \mid G x \leq g\right\}, \\
& u_{k} \in \mathbb{U} \triangleq\left\{u \in \mathbb{R}^{m} \mid H u \leq h\right\},
\end{aligned}
$$

for all $k$, where $G \in \mathbb{R}^{r_{x} \times n}, g \in \mathbb{R}^{r_{x}}, H \in \mathbb{R}^{r_{u} \times m}, h \in \mathbb{R}^{r_{u}}$, being $r_{x} \in \mathbb{Z}_{+}$and $r_{u} \in \mathbb{Z}_{+}$the number of state and input constraints, respectively.

Note that in (1b) a subset of controlled flows are directly related with a subset of uncontrolled flows. Hence, $u$ does not take values in $\mathbb{R}^{m}$ but in a linear variety. This latter observation, in addition to Assumption 2 , can be exploited to develop an affine parametrization of control variables in terms of a minimum set of disturbances as shown in [21, Appendix A], mapping control problems to a space with a smaller decision 
vector and with less computational burden due to the elimination of the equality constraints. Thus, the system (1) can be rewritten as

$$
x_{k+1}=A x_{k}+\tilde{B} \tilde{u}_{k}+\tilde{B}_{d} d_{k},
$$

and the input constraint $(3 \mathrm{~b})$ replaced with a time-varying restricted set defined as

$$
\tilde{\mathbb{U}}_{k} \triangleq\left\{\tilde{u} \in \mathbb{R}^{m-q} \mid H \tilde{P}_{1} \tilde{u} \leq h-H \tilde{P}_{2} d_{k}\right\} \quad \forall k \in \mathbb{Z}_{+},
$$

where $\tilde{B} \in \mathbb{R}^{n \times(m-q)}, \tilde{B}_{d} \in \mathbb{R}^{n \times p}, \tilde{P}_{1} \in \mathbb{R}^{m \times(m-q)}$ and $\tilde{P}_{2} \in \mathbb{R}^{m \times p}$, are selection and permutation matrices (see [21, Appendix A] for details). The sets $\tilde{\mathbb{U}}_{k}$ are non-empty for all $k$ due to Assumption 4.

\subsection{Control Problem Statement}

The goal is to design a control law that minimises a (possibly multi-objective) convex stage cost $\ell(k, x, \tilde{u})$ : $\mathbb{Z}_{+} \times \mathbb{X} \times \tilde{\mathbb{U}}_{k} \rightarrow \mathbb{R}_{+}$, which bears a functional relationship to the economics of the system operation. Let $x_{k} \in \mathbb{X}$ be the current state and let $\mathbf{d}_{k}=\left\{d_{k+i \mid k}\right\}_{i \in \mathbb{Z}_{[0, N-1]}}$ be the sequence of disturbances over a given prediction horizon $N \in \mathbb{Z}_{\geq 1}$. The first element of this sequence is measured, i.e., $d_{k \mid k}=d_{k}$, while the rest of the elements are estimates of future disturbances computed by an exogenous system and are available at each time step $k \in \mathbb{Z}_{+}$. Hence, the MPC controller design is based on the solution of the following finite-horizon optimization problem:

$$
\min _{\widetilde{\mathbf{u}}_{k}} \sum_{i=0}^{N-1} \ell\left(k+i, x_{k+i \mid k}, \tilde{u}_{k+i \mid k}\right)
$$

subject to:

$$
\begin{array}{ll}
x_{k+i+1 \mid k}=A x_{k+i \mid k}+\tilde{B} \tilde{u}_{k+i \mid k}+\tilde{B}_{d} d_{k+i \mid k}, & \forall i \in \mathbb{Z}_{[0, N-1]} \\
x_{k+i \mid k} \in \mathbb{X}, & \forall i \in \mathbb{Z}_{[1, N]} \\
\tilde{u}_{k+i \mid k} \in \tilde{\mathbb{U}}_{k+i}, & \forall i \in \mathbb{Z}_{[0, N-1]} \\
x_{k \mid k}=x_{k} . &
\end{array}
$$

Assuming that (6) is feasible, i.e., there exists a non-empty control input sequence $\tilde{\mathbf{u}}_{k}=\left\{\tilde{u}_{k+i \mid k}\right\}_{i \in \mathbb{Z}_{[0, N-1]}}$, then the receding horizon philosophy and the model back-transformation commands to apply the control input

$$
u_{k}=\kappa_{N}\left(k, x_{k}, \mathbf{d}_{k}\right)=\tilde{P}_{1} \tilde{u}_{k \mid k}^{*}+\tilde{P}_{2} d_{k} .
$$

This procedure is repeated at each time instant $k$, using the current measurements of states and disturbances and the most recent forecast of these latter over the next future horizon.

\section{SMPC approaches applied to DWN}

Given the stochastic nature of future disturbances, the prediction model (4) involves additive uncertainty, i.e., the water demand, which is an unmanipulated input. In this way, the change in water demand depends on the uncertainty of external factors, see, e.g., [32, 33]. Therefore, the historical water demand, in an implicit manner, takes into account historical data related to demographic, meteorological, and hydrological conditions, among other factors. In this sense, the compliance of constraints for a given control input cannot be ensured. Consequently, the use of SMPC strategies may allow to establish a trade-off between robustness and performance. In this context, three SMPC approaches are formulated below.

\subsection{CC-MPC}

Since the optimal solution to (6) does not always imply feasibility of the real system, it is appropriate to relax the original constraints in (21c) with probabilistic statements in the form of the so-called chance constraints. In this way, state constraints are required to be satisfied with a predefined probability to manage the reliability of the system. Considering the form of the state constraint set $\mathbb{X}$, there are two types of chance constraints according to the definitions below. 
Definition 1 (Joint chance constraint) A (linear) state joint chance constraint is of the form

$$
\mathbb{P}\left[G_{(j)} x \leq g_{(j)}, \forall j \in \mathbb{Z}_{\left[1, r_{x}\right]}\right] \geq 1-\delta_{x},
$$

where $\mathbb{P}$ denotes the probability operator, $\delta_{x} \in(0,1)$ is the risk acceptability level of constraint violation for the states, and $G_{(j)}$ and $g_{(j)}$ denote the $j^{\text {th }}$ row of $G$ and $g$, respectively. This requires that all rows $j$ have to be jointly fulfilled with the probability $1-\delta_{x}$.

Definition 2 (Individual chance constraint) A (linear) state individual chance constraint is of the form

$$
\mathbb{P}\left[G_{(j)} x \leq g_{(j)}\right] \geq 1-\delta_{x, j}, \quad \forall j \in \mathbb{Z}_{\left[1, r_{x}\right]},
$$

which requires that each $j^{\text {th }}$ row of the inequality has to be fulfilled individually with the respective probability $1-\delta_{x, j}$, where $\delta_{x, j} \in(0,1)$.

Both forms of constraints are useful to measure risks, hence, their selection depends on the application. All chance-constrained models require prior knowledge of the acceptable risk $\delta_{x}$ associated with the constraints. A lower risk acceptability implies a harder constraint. This article is concerned with the use of joint chance constraints since they can express better the management of the overall reliability in a DWN. In general, joint chance constraints lack from analytic expressions due to the involved multivariate probability distribution. Nevertheless, sampling-based methods, numeric integration, and convex analytic approximations exists, see e.g., [15] and references therein. Here, (8) is approximated following the results in [34, 35] by upper bounding the joint constraint and assuming a uniform distribution of the joint risk among a set of individual chance constraints that are later transformed into equivalent deterministic constraints under Assumption 5.

Assumption 5 Each demand in $d \in \mathbb{R}^{p}$ follows a log-concave univariate distribution, whose stochastic description is known.

Given the dynamic model in (4), the stochastic nature of the demand vector $d$ makes the state vector $x \in \mathbb{R}^{n}$ to be also a stochastic variable. Then, let the cumulative distribution function of the constraint be denoted as

$$
F_{G x}(g) \triangleq \mathbb{P}\left[\left\{G_{(1)} x \leq g_{(1)}, \ldots, G_{\left(r_{x}\right)} x \leq g_{\left(r_{x}\right)}\right\}\right] .
$$

Defining the events $C_{j} \triangleq\left\{G_{(j)} x \leq g_{(j)}\right\}$ for all $j \in \mathbb{Z}_{1}^{r_{x}}$, and denoting their complements as $C_{j}^{c} \triangleq\left\{G_{(j)} x>g_{(j)}\right\}$, then it follows that

$$
\begin{aligned}
F_{G x}(g) & =\mathbb{P}\left[C_{1} \cap \ldots \cap C_{r_{x}}\right] \\
& =\mathbb{P}\left[\left(C_{1}^{c} \cup \ldots \cup C_{r_{x}}^{c}\right)^{c}\right] \\
& =1-\mathbb{P}\left[\left(C_{1}^{c} \cup \ldots \cup C_{r_{x}}^{c}\right)\right] \geq 1-\delta_{x} .
\end{aligned}
$$

Taking advantage of the union bound, the Boole's inequality allows to bound the probability of the second term in the left-hand side of (11c), stating that for a countable set of events, the probability that at least one event happens is not higher than the sum of the individual probabilities [34]. This yields

$$
\mathbb{P}\left[\bigcup_{j=1}^{r_{x}} C_{j}^{c}\right] \leq \sum_{j=1}^{r_{x}} \mathbb{P}\left[C_{j}^{c}\right] .
$$

Applying (12) to the inequality in (11c), it follows that

$$
\sum_{j=1}^{r_{x}} \mathbb{P}\left[C_{j}^{c}\right] \leq \delta_{x} \Leftrightarrow \sum_{j=1}^{r_{x}}\left(1-\mathbb{P}\left[C_{j}\right]\right) \leq \delta_{x}
$$

At this point, a set of constraints arises from previous result as sufficient conditions to enforce the joint chance constraint (8), by allocating the joint risk $\delta_{x}$ in separate individual risks denoted by $\delta_{x, j}, j \in \mathbb{Z}_{1}^{r_{x}}$. 
These constraints are

$$
\begin{aligned}
& \mathbb{P}\left[C_{j}\right] \geq 1-\delta_{x, j}, \quad \forall j \in \mathbb{Z}_{1}^{r_{x}}, \\
& \sum_{j=1}^{r_{x}} \delta_{x, j} \leq \delta_{x}, \\
& 0 \leq \delta_{x, j} \leq 1,
\end{aligned}
$$

where (14) forms the set of $r_{x}$ resultant individual chance constraints, which bounds the probability that each inequality of the receding horizon problem may fail; and (15) and (16) are conditions imposed to bound the new single risks in such a way that the joint risk bound is not violated. Any solution that satisfies the above constraints, is guaranteed to satisfy (8). As proposed in [35], assigning, e.g., a fixed and equal value of risk to each individual constraint, i.e., $\delta_{x, j}=\delta_{x} / r_{x}$ for all $j \in \mathbb{Z}_{\left[1, r_{x}\right]}$, then (15) and (16) are satisfied.

Remark 1 The single risks $\delta_{x, j}, j \in \mathbb{Z}_{\left[1, r_{x}\right]}$, might be considered as new decision variables to be optimised, see e.g., [36]. This should improve the performance but at the cost of more computational burden due to the greater complexity and dimensionality of the optimization task. Therefore, as DWNs are often large-scale systems, the uniform risk allocation policy is adopted to avoid overloading of the optimization problem.

After decomposing the joint constraints into a set of individual constraints, the deterministic equivalent of each separate constraint may be used given that the probabilistic statements are not suitable for algebraic solution. Such deterministic equivalents might be obtained following the results in [37]. Assuming a known (or approximated) quasi-concave probabilistic distribution function for the effect of the stochastic disturbance in the dynamic model (4), it follows that

$$
\begin{aligned}
\mathbb{P}\left[G_{(j)} x_{k+1} \leq g_{(j)}\right] \geq 1-\delta_{x, j} & \Leftrightarrow F_{G_{(j)} \tilde{B}_{d} d_{k}}\left(g_{(j)}-G_{(j)}\left(A x_{k}+\tilde{B} \tilde{u}_{k}\right)\right) \geq 1-\delta_{x, j} \\
& \Leftrightarrow G_{(j)}\left(A x_{k}+\tilde{B} \tilde{u}_{k}\right) \leq g_{(j)}-F_{G_{(j)} \tilde{B}_{d} d_{k}}^{-1}\left(1-\delta_{x, j}\right),
\end{aligned}
$$

for all $j \in \mathbb{Z}_{\left[1, r_{x}\right]}$, where $F_{G_{(j)} \tilde{B}_{d} d_{k}}(\cdot)$ and $F_{G_{(j)} \tilde{B}_{d} d_{k}}^{-1}(\cdot)$ are the cumulative distribution and the left-quantile function of $G_{(j)} \tilde{B}_{d} d_{k}$, respectively. Hence, the original state constraint set $\mathbb{X}$ is contracted by the effect of the $r_{x}$ deterministic equivalents in (17) and replaced by the stochastic feasibility set given by

$$
\begin{aligned}
& \mathbb{X}_{s, k} \triangleq\left\{x_{k} \in \mathbb{R}^{n} \mid \exists \tilde{u}_{k} \in \overline{\mathbb{U}}_{k},\right. \text { such that } \\
& \left.\qquad G_{(j)}\left(A x_{k}+\tilde{B} \tilde{u}_{k}\right) \leq g_{(j)}-F_{G_{(j)} \tilde{B}_{d} d_{k}}^{-1}\left(1-\delta_{x, j}\right), \forall j \in \mathbb{Z}_{\left[1, r_{x}\right]}\right\},
\end{aligned}
$$

for all $k \in \mathbb{Z}_{+}$. From convexity of $G_{(j)} x_{k+1} \leq g_{(j)}$ and Assumption 5 , it follows that the set $\mathbb{X}_{s, k}$ is convex when non-empty for all $\delta_{x, j} \in(0,1)[38]$ and most quasi-concave distribution function. For some particular distributions, e.g., Gaussian, convexity is retained for $\delta_{x, j} \in(0,0.5]$

In this way, the reformulated predictive controller solves the following deterministic equivalent optimization problem for the expectation $\mathbb{E}[\cdot]$ of the cost function in (6a):

$$
\min _{\tilde{\mathbf{u}}_{k}} \sum_{i=0}^{N-1} \mathbb{E}\left[\ell\left(k+i, x_{k+i \mid k}, \tilde{u}_{k+i \mid k}\right)\right],
$$

subject to:

$$
\begin{array}{ll}
x_{k+i+1 \mid k}=A x_{k+i \mid k}+\tilde{B} \tilde{u}_{k+i \mid k}+\tilde{B}_{d} d_{k+i \mid k}, & \forall i \in \mathbb{Z}_{[0, N-1]} \\
G_{(j)}\left(A x_{k+i \mid k}+\tilde{B} \tilde{u}_{k+i \mid k}\right) \leq g_{(j)}-z_{k, j}\left(\delta_{x}\right), & \forall i \in \mathbb{Z}_{[0, N-1]}, \forall j \in \mathbb{Z}_{\left[1, r_{x}\right]}, \\
\tilde{u}_{k+i \mid k} \in \tilde{\mathbb{U}}_{k+i}, & \forall i \in \mathbb{Z}_{[0, N-1]} \\
x_{k \mid k}=x_{k}, &
\end{array}
$$

where $\tilde{\mathbf{u}}_{k}=\left\{\tilde{u}_{k+i \mid k}\right\}_{i \in \mathbb{Z}_{[0, N-1]}}$ is the sequence of controlled flows, $d_{k \mid k}=d_{k}$ is the current demand and $d_{k+i \mid k}$ are expected future demands computed at time instant $k \in \mathbb{Z}_{+}$for $i$-steps ahead with $i \in \mathbb{Z}_{[1, N-1]}$. Moreover, 

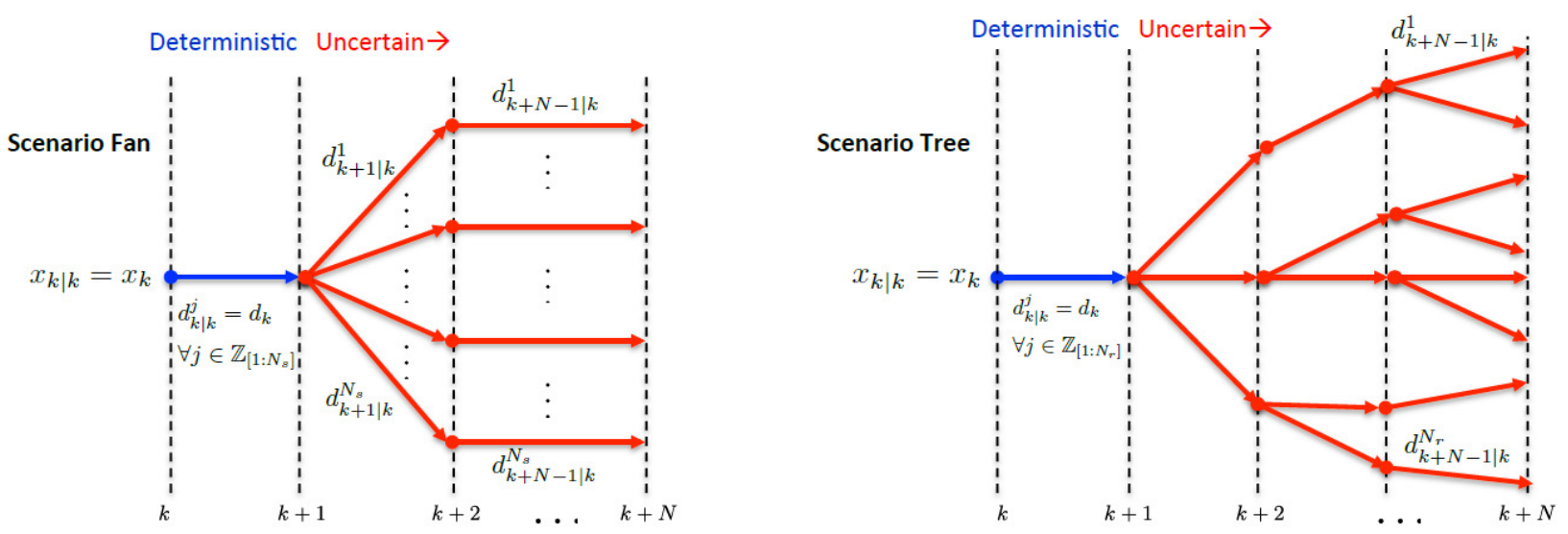

Figure 3: Reduction of a disturbance fan (left) of equally probable scenarios into a rooted scenario-tree (right).

$z_{k, j}\left(\delta_{x}\right) \triangleq F_{G_{(j)} \tilde{B}_{d} d_{k+i}}^{-1}\left(1-\frac{\delta_{x}}{n_{c}}\right)$, where $n_{c} \in \mathbb{Z}_{\geq 1}$ is the number of total individual state constraints along the prediction horizon, i.e., $n_{c}=r_{x} N$. Since $n_{c}$ depends not only on the number of state constraints $r_{x}$ but also on the value of $N$, the decomposition of the original joint chance constraint within the MPC algorithm could lead to a large number of constraints. This fact reinforces the use of a fixed risk distribution policy for DWN control problems, in order to avoid the addition of a large number of new decision variables to be optimized.

Remark 2 It turns out that most (not all) probability distribution functions used in different applications, e.g., uniform, Gaussian, logistic, Chi-squared, Gamma, Beta, log-normal, Weibull, Dirichlet, Wishart, among others, share the property of being log-concave. Then, their corresponding quantile function can be computed off-line for a given risk acceptability level and used within the MPC convex optimization.

\subsection{TB-MPC}

CC-MPC might be conservative if the probabilistic distributions of the stochastic variables are not properly characterized or do not have a log-concave form. Therefore, this section presents the TB-MPC strategy that relies on scenario trees to approximate the original problem, dropping Assumption 5. The approach followed by TB-MPC is based on modelling the possible scenarios of the disturbances as a rooted tree, as seen in Fig. 3. This means that all the scenarios start from the same measured disturbance value. From that initial point, the scenarios must remain equal until the point in which they diverge behaving differently. This point is called a bifurcation point, as described in [27]. Each node of the tree has a unique parent and can have many children. The total number of children at the last stage corresponds to the total number of scenarios. The probability of a scenario is the product of probabilities of each node in that scenario. Recall that a large number of scenarios might improve the robustness of the TB-MPC approach but at the cost of additional computational burden and economic performance losses. Hence, a trade-off must be achieved between performance and computational burden.

Notice that before a bifurcation point, the evolution followed by the disturbance cannot be anticipated because different evolutions are possible. For this reason, the controller has to calculate control inputs that are valid for all the scenarios in the branch. Once the bifurcation point has been reached, the uncertainty is solved and the controller can calculate specific control inputs for the scenarios in each of the new branches [39]. Hence, the outcome of TB-MPC is not a single sequence of control inputs, it is a tree with the same structure of that of the disturbances. Only the first element of this tree is applied (the root) and the problem is repeated in a receding horizon fashion.

The easiest way to understand the optimization problem associated to TB-MPC is to solve as many instances of Problem (6) as the number $N_{r} \in \mathbb{Z}_{+}$of scenarios considered, but formally it is a multi-stage stochastic programming problem that should be and solved as a big optimization problem for all the scenarios. Due to the increasing uncertainty, it is necessary to include non-anticipativity constraints [40] into the MPC 
formulation so that the calculated input sequence is always ready to face any possible future bifurcation in the tree [41]. TB-MPC constrains the control inputs relative to a scenario and its parent from the root at time instant $k$ to the branching point. In this sense, it is necessary to define the tree structure, at each time step $k$ over the horizon $N$, by means of two functions for each scenario: a parent function $P(\cdot)$, defined from $\mathbb{Z}_{\left[1, N_{r}\right]}$ to $\mathbb{Z}_{\left[1, N_{r}\right]}$, which indicates from which scenario it branches out; and a branching point function $B_{P}(\cdot)$, defined from $\mathbb{Z}_{\left[1, N_{r}\right]}$ to $\mathbb{Z}_{[k, k+N]}$, which indicates the time when the scenario emerges as different from its parent. For more details, see [27] and references therein.

More specifically, if $\mathbf{d}_{k}^{a}=\left\{d_{k \mid k}^{a}, d_{k+1 \mid k}^{a}, \ldots, d_{k+N \mid k}^{a}\right\}$ and $\mathbf{d}_{k}^{b}=\left\{d_{k \mid k}^{b}, d_{k+1 \mid k}^{b}, \ldots, d_{k+N \mid k}^{b}\right\}$ are two disturbance sequences corresponding respectively to the forecast scenarios $a, b \in \mathbb{Z}_{\left[1, N_{r}\right]}$, then the non-anticipative constraint $\tilde{u}_{k+i \mid k}^{a}=\tilde{u}_{k+i \mid k}^{b}$ must be satisfied whenever the parent of the scenario $a$ was $b$, that is $P(a)=b$, and the scenario $a$ has not emerged yet at time instant $k+i$, i.e., $k+i<B_{P}(a)$ for any $i \in \mathbb{Z}_{[0, N]}$ in order to guarantee that for all $j \in \mathbb{Z}_{\left[1, N_{r}\right]}$ the control input sequences $\tilde{\mathbf{u}}^{j}=\left\{\tilde{u}_{k+i \mid k}^{j}\right\}_{i \in \mathbb{Z}_{[0, N-1]}}$ do not depend on the unobserved stochastic variables, as established in [22].

In this way, the TB-MPC controller should solve the following optimization problem at each time instant $k \in \mathbb{Z}_{+}$, accounting for the $N_{r}$ demand scenarios, each with probability $p_{j} \in(0,1]$ satisfying $\sum_{j=1}^{N_{r}} p_{j}=1$ :

$$
\min _{\tilde{\mathbf{u}}_{k}^{j}} \sum_{j=1}^{N_{r}} p_{j}\left(\sum_{i=0}^{N-1} \ell\left(k+i, x_{k+i \mid k}^{j}, \tilde{u}_{k+i \mid k}^{j}\right)\right),
$$

subject to:

$$
\begin{aligned}
& x_{k+i+1 \mid k}^{j}=A x_{k+i \mid k}^{j}+\tilde{B} \tilde{u}_{k+i \mid k}^{j}+\tilde{B}_{d} d_{k+i \mid k}^{j}, \\
& x_{k+i+1 \mid k}^{j} \in \mathbb{X}, \\
& \tilde{u}_{k+i \mid k}^{j} \in \tilde{\mathbb{U}}_{k+i}^{j}, \\
& x_{k \mid k}^{j}=x_{k}, \quad d_{k \mid k}^{j}=d_{k}, \\
& \tilde{u}_{k+i \mid k}^{a}=\tilde{u}_{k+i \mid k}^{b} \text { when }\left\{\begin{array}{l}
P(a)=b, \\
k+i<B_{P}(a)
\end{array} \quad \forall a, b \in \mathbb{Z}_{\left[1, N_{r}\right]},\right.
\end{aligned}
$$

for all $i \in \mathbb{Z}_{[0, N-1]}$ and all $j \in \mathbb{Z}_{\left[1, N_{r}\right]}$, where $\tilde{\mathbb{U}}_{k+i}^{j} \triangleq\left\{\tilde{u}^{j} \in \mathbb{R}^{m-q} \mid H \tilde{P} \tilde{M}_{1} \tilde{u}^{j} \leq h-H \tilde{P} \tilde{M}_{2} d_{k+i}^{j}\right\}$.

Remark 3 In practice, Problem (19) can be solved more efficiently by using a transformation matrix that removes the redundancy of the optimization variables due to non-anticipative constraints (19f). In this manner, the number of optimization variables and constraints decrease [42].

The control signal is the same for all scenarios at the current time step since these scenarios start with the measured disturbance and equality constraints are formulated. The optimization problem meets these constraints and computes the control input under economic policies. If the solver is not able to calculate the control inputs by infeasibility, these equality constraints can become soft-constraints and penalize deviations. In addition, note that uncertainty spreads with time, i.e., the estimation of the disturbances at the first time instant can be carried out with a certain degree of accuracy. Scenarios tend to diverge with time because the uncertainty grows as well.

Remark 4 Another way to solve the optimization problem in (19) is to treat each scenario independently by considering distributed optimization and attain a coordinated solution using a distributed control scheme, as shown in [41].

\subsection{MS-MPC}

The optimization based on scenarios provides an intuitive way to approximate the solution of the stochastic optimization problem. The MS-MPC approach can be implemented similarly to the TB-MPC. The difference between both approaches is that while TB-MPC calculates as many control sequences as scenarios considered, MS-MPC optimizes a single control sequence valid for all the scenarios. 
The MS-MPC approach is computationally efficient since its solution is based on a deterministic convex optimization, even when the original problem is not [43]. Thus, a stochastic optimization is solved in a deterministic way. One of its advantages is that it is possible to calculate the risk acceptability level ( $\left.\delta_{x}\right)$ of constraint violation as a function of the number of scenarios considered for any violation level $v \in[0,1][28]$. In particular, the probability of the predicted states to satisfy the constraints is given by

$$
\mathbb{P}\left[x_{i+1} \in \mathcal{X}, \quad \forall i \in \mathbb{Z}_{0}^{N-1}\right] \geq 1-\delta_{x},
$$

where

$$
\delta_{x}=\int_{0}^{1}\left[\min \left\{1,\left(\begin{array}{c}
R+\rho_{1}-1 \\
R
\end{array}\right) \sum_{j=0}^{R+\rho_{1}-1}\left(\begin{array}{c}
K \\
j
\end{array}\right) v^{j}(1-v)^{K-j}\right\} d v\right] .
$$

As it can be seen, the risk acceptability level $\delta_{x}$ is a function of $K \in \mathbb{Z}_{+}$that establishes the total number of scenarios generated that represent the evolutions of the water demand, $R \in \mathbb{Z}_{+}$that represents the number of discarded scenarios $(R<K)$, and $\rho_{1}$ which is a parameter related to the algebraic properties of the problem [43]. Notice that $N_{s}=K-R$, that is, the number of scenarios considered in the FHOP.

Remark 5 Note that the calculation of probabilistic bounds for TB-MPC is beyond the scope of this work. However, given that the performance of TB-MPC is better than that of $M S-M P C$ (the controller is executed in closed loop from a control viewpoint in TB-MPC), the same probabilistic bounds could be used for both, $M S-M P C$ and $T B-M P C$, at least as a practical rule.

The MS-MPC controller should solve the following FHOP, accounting for the $N_{s} \in \mathbb{Z}_{+}$scenarios:

$$
\min _{\tilde{\mathbf{u}}_{k}^{j}} \sum_{j=1}^{N_{r}} p_{j}\left(\sum_{i=0}^{N-1} \ell\left(k+i, x_{k+i \mid k}^{j}, \tilde{u}_{k+i \mid k}\right)\right),
$$

subject to:

$$
\begin{aligned}
& x_{k+i+1 \mid k}^{j}=A x_{k+i \mid k}^{j}+\tilde{B} \tilde{u}_{k+i \mid k}+\tilde{B}_{d} d_{k+i \mid k}^{j}, \\
& x_{k+i+1 \mid k}^{j} \in \mathbb{X}, \\
& \tilde{u}_{k+i \mid k} \in \tilde{\mathbb{U}}_{k+i}, \\
& x_{k \mid k}^{j}=x_{k}, \quad d_{k \mid k}^{j}=d_{k},
\end{aligned}
$$

for all $i \in \mathbb{Z}_{[0, N-1]}$ and all $j \in \mathbb{Z}_{\left[1, N_{s}\right]}$, where $p_{j}$ is again the probability of occurrence of each considered scenario [17].

It should be noticed that this approach reduces the number of decision variables in the FHOP compared to the TB-MPC approach, but it might increase the computational time since having less degrees of freedom increases the complexity of complying simultaneously with all the considered scenarios.

Remark 6 One way to address the complexity of the MS-MPC optimization problem is to follow the ideas presented in [17, 44], where only three disturbance scenarios are considered, i.e., the best, the worst and the average case, each one with its probability of occurrence. This is a practical way to relax the computational burden.

In this paper, the simplification highlighted in Remark 6 is adopted. In this way, the MS-MPC approach considers the solution of (21) with $N_{s}=3$.

\section{Results}

The formulation of the SMPC problems for the case studies considered in this paper addresses the design of a control law that (i) minimizes the economic operational cost, (ii) guarantees the availability of enough water to satisfy the demand and (iii) operates the network with smooth variations of the flow through actuators. These objectives can be expressed quantitatively by the following performance indicators ${ }^{2}$ for all time steps

\footnotetext{
${ }^{2}$ The performance indicators considered in this paper may vary or be generalized with the corresponding manipulation to include other control objectives.
} 
$k \in \mathbb{Z}_{+}:$

$$
\begin{aligned}
& \ell_{E}\left(x_{k}, \tilde{u}_{k} ; c_{u, k}\right) \triangleq c_{u, k}^{\top} W_{e} \tilde{u}_{k} \Delta t, \\
& \ell_{S}\left(x_{k} ; s_{k}\right) \triangleq \begin{cases}\left(x_{k}-s_{k}\right)^{\top} W_{s}\left(x_{k}-s_{k}\right) & \text { if } x_{k} \leq s_{k} \\
0 & \text { otherwise }\end{cases} \\
& \ell_{\Delta}\left(\Delta \tilde{u}_{k}\right) \triangleq \Delta \tilde{u}_{k}^{\top} W_{\Delta \tilde{u}} \Delta \tilde{u}_{k} .
\end{aligned}
$$

The first objective, $\ell_{E}\left(x_{k}, \tilde{u}_{k} ; c_{u, k}\right) \in \mathbb{R}_{\geq 0}$, represents the economic cost of network operation at time step $k$, which depends on a time-of-use pricing scheme driven by a time-varying price of the flow through the actuators $c_{u, k} \triangleq\left(c_{1}+c_{2, k}\right) \in \mathbb{R}_{+}^{m-q}$, which in this application takes into account a fixed water production cost $c_{1} \in \mathbb{R}_{+}^{m}$ and a water pumping cost $c_{2, k} \in \mathbb{R}_{+}^{m}$ that changes according to the electricity tariff (assumed periodically time-varying). All prices are given in economic units per cubic meter (e.u. $\left./ \mathrm{m}^{3}\right)$. The second objective, $\ell_{S}\left(x_{k} ; s_{k}\right) \in \mathbb{R}_{\geq 0}$ for all $k$, is a performance index that penalizes the amount of water volume going below a given safety threshold $s_{k} \in \mathbb{R}^{n}$ in $\mathrm{m}^{3}$, which is desired to be stored in tanks and satisfies the condition $x_{\min } \leq s_{k} \leq x_{\max }$. Note that this safety objective is a piecewise continuous function, but it can be redefined as $\ell_{S}\left(\xi_{k} ; x_{k}, s_{k}\right) \triangleq \xi_{k}^{\top} W_{s} \xi_{k}$, accompanied with two additional convex constraints, i.e., $x_{k} \geq s_{k}-\xi_{k}$ and $\xi_{k} \in \mathbb{R}_{+}^{n}$, for all $k$, being $\xi_{k}$ a slack variable. The minimal volume of water required in a tank is given by its net demand, hence, $s_{k}=-B_{p} d_{k}$ for all $k$. The last objective, $\ell_{\Delta}\left(\Delta \tilde{u}_{k}\right) \in \mathbb{R}_{\geq 0}$, represents the penalization of control signal variations $\Delta \tilde{u}_{k} \triangleq \tilde{u}_{k}-\tilde{u}_{k-1} \in \mathbb{R}^{m-q}$. The inclusion of this latter objective aims to extend actuators life and assure a smooth operation of the dynamic network flows. Furthermore, $W_{e} \in \mathbb{S}_{++}^{m-q}, W_{s} \in \mathbb{S}_{++}^{n}$ and $W_{\Delta \tilde{u}} \in \mathbb{S}_{++}^{m-q}$ are matrices that weight each decision variable in their corresponding cost function.

To achieve the control task, the above predefined objectives are aggregated in a multi-objective stage cost function, which depends explicitly on time due to the time-varying parameters of the involved individual objectives. The overall stage cost is defined for all $k \in \mathbb{Z}_{+}$as

$$
\ell\left(k, x_{k}, \tilde{u}_{k}, \xi_{k}\right) \triangleq \lambda_{1} \ell_{E}\left(x_{k}, \tilde{u}_{k} ; c_{u, k}\right)+\lambda_{2} \ell_{\Delta}\left(\Delta \tilde{u}_{k}\right)+\lambda_{3} \ell_{S}\left(\xi_{k} ; x_{k}, s_{k}\right),
$$

where $\lambda_{1}, \lambda_{2}, \lambda_{3} \in \mathbb{R}_{+}$are scalar weights that allow to prioritize the impact of each objective involved in the overall performance of the network. These weights are assumed to be fixed by the managers of the DWN.

Numerical results of applying the three different SMPC approaches (CC-MPC, TB-MPC and MS-MPC) to the Barcelona DWN case studies are summarized in Tables 1, 2 and 3.

Simulations have been carried out over a time horizon of eight days, i.e., $n_{s}=192$ hours, with a sampling time of one hour. The patterns of the water demand in this paper were synthesized from real values measured in the considered demand of the Barcelona DWN between July $23^{\text {th }}$ and July $27^{\text {th }}$, 2007. Initial conditions, i.e., source capacities, initial volume of water at tanks and starting demands, are set a priori according to real data. The weights of the cost function (23) are $\lambda_{1}=100, \lambda_{2}=10$, and $\lambda_{3}=1$; these values allow to prioritize the impact of each objective involved in the overall performance of the network. The prediction horizon is selected as $N=24$ hours, due to the periodicity of disturbances. The formulation of the optimization problems and the closed-loop simulations have been carried out using MATLAB R2012a (64 bits) and CPLEX solver, running in a PC Core i7 at $3.2 \mathrm{GHz}$ with $16 \mathrm{~GB}$ of RAM.

The key performance indicators used to assess the aforementioned controllers are defined as follows:

$$
\begin{aligned}
& \Phi_{1} \triangleq \frac{24}{n_{s}} \sum_{k=1}^{n_{s}} \ell_{k} \\
& \Phi_{2} \triangleq\left|\left\{k \in \mathbb{Z}_{\left[1, n_{s}\right]} \mid x_{k}<-B_{p} d_{k}\right\}\right|, \\
& \Phi_{3} \triangleq \sum_{k=1}^{n_{s}} \sum_{i=1}^{n_{x}} \max \left\{0,-B_{p(i)} d_{k}-x_{k(i)}\right\}, \\
& \Phi_{4} \triangleq \frac{1}{n_{s}} \sum_{k=1}^{n_{s}} t_{k},
\end{aligned}
$$

where $\Phi_{1}$ is the average daily multi-objective cost with $\ell_{k}$ given by (23), $\Phi_{2}$ is the number of time instants where water demands are not satisfied, $\Phi_{3}$ is the accumulated volume of water demand that was not satisfied 
Table 1: Comparison of the CC-MPC, TB-MPC and MS-MPC applied to the sector model of the DWN.

\begin{tabular}{|c|c|c|c|c|c|c|c|c|c|c|c|c|}
\hline \multicolumn{4}{|c|}{ CC-MPC } & \multicolumn{5}{|c|}{ TB-MPC } & \multicolumn{4}{|c|}{ MS-MPC } \\
\hline$\Phi_{1}$ & $\Phi_{2}$ & $\Phi_{3}$ & $\Phi_{4}$ & $\Phi_{1}$ & $\Phi_{2}$ & $\Phi_{3}$ & $\Phi_{4}$ & $N_{r}$ & $\Phi_{1}$ & $\Phi_{2} \Phi_{3}$ & $\Phi_{4}$ & $N_{s}$ \\
\hline 0.358535 .80 & 0 & 0 & 0.0919 & $\mid \begin{array}{l}58397.14 \\
58515.40 \\
58589.15\end{array}$ & $\begin{array}{l}0 \\
1 \\
1\end{array}$ & $\begin{array}{c}0 \\
0.4813 \\
4.155\end{array}$ & $\begin{array}{l}1.2548 \\
1.9701 \\
3.0145\end{array}$ & \begin{tabular}{c|}
5 \\
10 \\
14
\end{tabular} & 60831.33 & 0 & 16.1547 & 1070.3 \\
\hline 0.258541 .19 & 0 & 0 & 0.707 & \begin{tabular}{|l|}
58515.37 \\
58678.12 \\
58647.27
\end{tabular} & $\begin{array}{l}0 \\
2 \\
4\end{array}$ & $\begin{array}{c}0 \\
0.7443 \\
4.0329\end{array}$ & $\begin{array}{c}3.2218 \\
7.5362 \\
27.0914\end{array}$ & $\begin{array}{l}19 \\
38 \\
75\end{array}$ & 69342.48 & 0 & 17.2401 & 1290.2 \\
\hline 0.158558 .29 & 0 & 0 & 0.716 & \begin{tabular}{|l}
58705.28 \\
58713.15 \\
58761.98
\end{tabular} & $\begin{array}{l}1 \\
0 \\
0\end{array}$ & $\begin{array}{c}0.2136 \\
0 \\
0\end{array}$ & $\begin{array}{l}35.6572 \\
42.7024 \\
54.4587\end{array}$ & $\begin{array}{l}107 \\
129 \\
149\end{array}$ & 66011.29 & 0 & 21.7521 & 1490.1 \\
\hline
\end{tabular}

over the simulation horizon $n_{s}$, and $\Phi_{4}$ is the average time in seconds required to solve the MPC problem at each time instant $k \in \mathbb{Z}_{\left[1, n_{s}\right]}$.

The effect of considering different levels of joint risk acceptability was analysed for the CC-MPC approach using $\delta=\{0.3,0.2,0.1\}$. In the same way, the size of the set of scenarios selected for the MS-MPC is established by using (20) to guarantee the same risk levels of the CC-MPC approach. In this way, the total number of scenarios that represents the evolution of the water demand in the considered simulation time for the MS-MPC was $K=192$. Likewise, the TB-MPC approach was applied considering different sizes for the set of reduced scenarios, i.e., $N_{r}=\{5,10,19,38,75,107,129,149\}$. The last three $N_{r}$ scenarios allow to compare the behaviour between MS-MPC and TB-MPC, while the remaining scenarios were used to analyse the performance with respect to a small number of scenarios.

Table 1 summarizes the results of applying the SMPC approaches to the sector model of the Barcelona DWN presented in Fig. 1. The different values of $\delta$ in the CC-MPC approach highlight that both reliability and control performance are conflicting objectives, i.e., the inclusion of safety mechanisms in the controller increases the reliability of the DWN in terms of demand satisfaction, but also the cost of its operation. The main advantage of the CC-MPC is its formal methodology, which leads to obtain optimal safety constraints that tackle uncertainties and allow to achieve a specified global service level in the DWN. Moreover, the CC-MPC robustness is achieved with a low computational burden given that the only extra load is the computation of the stochastic characteristics of disturbances propagated along the prediction horizon $N$. In this way, the CC-MPC approach is suitable for real-time control of large-scale DWNs.

Regarding the TB-MPC and MS-MPC approaches, numerical results show that considering a larger number of scenarios increments (in average) the stage cost while reducing the volume of unsatisfied water demand. This might be influenced by the quality of the information that remains after the reduction algorithms and, consequently, it affects the robustness of the approach being subject of further research.

The main drawback of the TB-MPC approach is the solution average time and the computational burden. The implementation for all cases taking scenarios greater than $N_{r}=149$ was not possible due to memory issues. Hence, some simplification assumptions as those used in [24] or parallel computing techniques might be useful. Another way to address the problem generated by the computer effort is to use a MS-MPC based on the three-scenarios approach, described in Subsection 4.3. At this point, the best, the worst and the average disturbance scenarios were obtained by generating 100 different possible evolutions of the disturbance, then they were lumped and averaged the 10 lowest, 10 highest, and 80 middle, respectively. It means that the occurrence probabilities were established as $0.1,0.1$, and 0.8 for the best, the worst, and the average disturbance scenario, respectively, as proposed in [17].

Additionally, Table 2 summarizes the simulation results of applying the studied SMPC approaches again to the sector model DWN. The configuration of the controllers in this case is as follows: the CC-MPC with a probability of risk of $5 \%$, the TB-MPC reducing to $N_{r}=3$ branched disturbances, the MS-MPC based on the three scenarios approach (i.e., best, worst and average), and the CE-MPC with an average disturbance. On the one hand, the CE-MPC approach presents the lower cost but on the other hand it has problems with the demand satisfaction. The TB-MPC approach presents a lower accumulated volume of unsatisfied 
water demand compared with respect to the CE-MPC approach. The MS-MPC and CC-MPC approaches are able to satisfy the water demand required by the consumers. The CC-MPC approach presents a better performance regarding cost and computational time compared to the MS-MPC approach.

Table 2: Comparison of the MS-MPC, TB-MPC, CC-MPC and CE-MPC applied to the sector model DWN.

\begin{tabular}{ccccc}
\hline Controller & $\Phi_{1}$ & $\Phi_{2}$ & $\Phi_{3}$ & $\Phi_{4}$ \\
\hline CC-MPC & 585401.16 & 0 & 0 & 0.1069 \\
TB-MPC & 58425.59 & 1 & 1.2229 & 1.4235 \\
MS-MPC & 60567.62 & 1 & 0.6965 & 0.5314 \\
CE-MPC & 58327.55 & 1 & 0.7411 & 0.1041 \\
\hline
\end{tabular}

As for the second case study, Table 3 presents the results obtained after the application of the SMPC approaches to the aggregate model DWN, as a way to show the strengths and weaknesses of each of the aforementioned approaches applied to a larger system. For this reason, TB-MPC and MS-MPC with a large number of scenarios, could not be applied due to memory issues. TB-MPC was implemented with a reduction to $N_{r}=3$ branched scenarios. MS-MPC has been designed considering the three scenarios (minimum, average and maximum) as explained in the previous case study. CC-MPC is applied to this system with a risk probability of $5 \%$. As it can be seen from the results, the TB-MPC approach does not offer benefits in terms of satisfaction of water demand and computational time with this limited amount of scenarios for the DWN aggregate model. MS-MPC presents encouraging results regarding demand satisfaction and computational time well below that TB-MPC. MS-MPC approach presents a higher average daily multiobjective cost and a computational time required to solve the FHOP around three times more regarding CC-MPC. Furthermore, MS-MPC and CC-MPC, have a good performance with respect to water demand satisfaction. Based on the obtained results, the CC-MPC approach offers better performance in terms of demand satisfaction, computational time and, it presents the best behaviour with respect to the average daily multi-objective cost $\left(\phi_{1}\right)$ compared with the same indicator obtained with TB-MPC and MS-MPC approaches.

Table 3: Comparison of the MS-MPC, TB-MPC, CC-MPC and CE-MPC applied to the DWN case study for the aggregate DWN model.

\begin{tabular}{ccccc}
\hline Controller & $\Phi_{1}$ & $\Phi_{2}$ & $\Phi_{3}$ & $\Phi_{4}$ \\
\hline CC-MPC & $1.4064 \cdot 10^{4}$ & 0 & 0 & 0.9056 \\
TB-MPC & $1.4497 \cdot 10^{4}$ & 20 & 18.81 & 8.48 \\
MS-MPC & $1.5267 \cdot 10^{4}$ & 0 & 0 & 3.24 \\
CE-MPC & $1.2038 \cdot 10^{4}$ & 23 & 5.211 & 0.8442 \\
\hline
\end{tabular}

As expected, the SMPC approaches have a better performance indicators with respect to CE-MPC approach, which does not take into account the stochastic nature of water demand in the formulation of FHOP.

\section{Conclusions}

In this paper, three stochastic control approaches have been assessed to deal with the optimal operational management of a DWN. The proposed stochastic control strategies are based on MPC which allows the optimization of the objective function to ensure a reliable water supply in the most economic and safe way by considering the uncertainty in the water consumption. Simulation results show that all the considered methods require less than 1 minute to solve the optimization problem in each case, much shorter than the sampling time of 1 hour. Hence, it is possible to choose an approach that shows the best performance in terms of demand satisfaction, which is given by the number of time instants where water demands were not satisfied and by the cumulated volume of non-satisfied water demand. In this sense, the results in this paper show that CC-MPC is more appropriate when requiring a low probability of constraint violation, because 
the use of TB-MPC and MS-MPC implies the inclusion of a higher number of scenarios, which hinders the application of these control strategies to large DWNs. However, the use of these scenarios-based approaches may be very demanding in terms of computational time.

From a practical viewpoint, both TB-MPC and CC-MPC outperform MS-MPC. TB-MPC excels due to its close-loop control capability and requires to build a tree to reproduce the main dynamics of the disturbance $[27,45,46]$. CC-MPC improves the performance by allowing a probability of constraint violation and requires considering a well-known behaviour of water user demand in terms of probability distribution. Finally, MS-MPC is an alternative to cope with the uncertainty, which considers the possible evolutions of the water demand modelled as scenarios, by using an open-loop forecasting of disturbances. A drawback that presents this approach is its reduced capacity to be adapted to changes that the water demand could arise. This may result in more conservative control inputs that increase the economic costs.

Each of the approaches described have their advantages and weakness. CC-MPC presents the best computational time and economic costs against the need to take account of a probability distribution function that represents the behaviour of the disturbances. TB-MPC and MS-MPC approaches are ways to solve the stochastic optimization in a deterministic manner, but require a greater computational effort. Finally TB-MPC and MS-MPC approaches can be implemented using a reduced number of scenarios at the cost of lower robustness.

\section{References}

[1] Alegre H, Baptista J, Cabrera E, Cubillo F. Performance Indicators for Water Supply Services. Manuals of Best Practice Series, IWA Publishing, 2006.

[2] Ocampo-Martinez C, Puig V, Cembrano G, Quevedo J. Application of predictive control strategies to the management of complex networks in the urban water cycle. Control Systems, IEEE 2013; 33(1):15-41.

[3] Camacho EF, Bordons C. Model Predictive Control in the Process Industry. Second Edition. SpringerVerlag: London, England, 2004.

[4] Maciejowski J. Predictive control with constraints. Prentice Hall: Essex, England, 2002.

[5] Biscos C, Mulholland M, Le Lann M, Buckley C, Brouckaert C. Optimal operation of water distribution networks by predictive control using MINLP. Water Sa 2004; 29(4):393-404.

[6] Leirens S, Zamora C, Negenborn R, De Schutter B. Coordination in urban water supply networks using distributed model predictive control. Proceedings of the American Control Conference (ACC), Baltimore, Maryland, USA, 2010; 3957-3962.

[7] Grosso J, Ocampo-Martinez C, Puig V. A service reliability model predictive control with dynamic safety stocks and actuators health monitoring for drinking water networks. Proceedings of 51st IEEE Annual Conference on Decision and Control (CDC), Maui, Hawaii, USA, 2012; 4568-4573.

[8] Zafra-Cabeza A, Maestre JM, Ridao MA, Camacho EF, Sánchez L. A hierarchical distributed model predictive control approach in irrigation canals: A risk mitigation perspective. Journal of Process Control 2011; $21(5): 787-799$.

[9] van Overloop PJ, Van Clemmens AJ, Strand RJ, Wagemaker R, Bautista E. Real-time implementation of model predictive control on Maricopa-Stanfield irrigation and drainage district's WM canal. Journal of Irrigation and Drainage Engineering 2010; 136(11):747-756.

[10] van de Water $\mathrm{H}$, Willems J. The certainty equivalence property in stochastic control theory. IEEE Transactions on Automatic Control oct 1981; 26(5):1080 - 1087.

[11] van de Water $\mathrm{H}$, Willems J. The certainty equivalence property in stochastic control theory. IEEE Transactions on Automatic Control 2002; 26(5):1080-1087. 
[12] Bernardini D, Bemporad A. Scenario-based model predictive control of stochastic constrained linear systems. Proceedings of the 48th IEEE Conference on Decision and Control (CDC), held jointly with the 28th Chinese Control Conference (CCC), Shanghai, China, 2009; 6333-6338.

[13] Cannon M, Couchman P, Kouvaritakis B. MPC for stochastic systems. Assessment and Future Directions of Nonlinear Model Predictive Control, Lecture Notes in Control and Information Sciences, vol. 358, Findeisen R, Allgöwer F, Biegler L (eds.). Springer Berlin Heidelberg, 2007; 255-268.

[14] Muñoz de la Peña D, Bemporad A, Alamo T. Stochastic programming applied to model predictive control. Proceedings of 44th IEEE Conference on Decision and Control and European Control Conference (CDC-ECC), Seville, 2005; $1361-1366$.

[15] Calafiore G, Dabbene F. Probabilistic and randomized methods for design under uncertainty. Springer: London, England, 2006.

[16] Calafiore G, Dabbene F, Tempo R. Research on probabilistic methods for control system design. Automatica 2011; 47(7):1279 - 1293.

[17] van Overloop PJ, Weijs S, Dijkstra S. Multiple model predictive control on a drainage canal system. Control Engineering Practice 2008; 16(5):531 - 540.

[18] Schwarm A, Nikolaou M. Chance-constrained model predictive control. AIChE Journal 1999; 45(8):1743-1752.

[19] Geletu A, Klöppel M, Zhang H, Li P. Advances and applications of chance-constrained approaches to systems optimisation under uncertainty. International Journal of Systems Science 2013; 44(7):12091232 .

[20] Ouarda T, Labadie J. Chance-constrained optimal control for multireservoir system optimization and risk analysis. Stochastic Environmental Research and Risk Assessment 2001; 15:185-204.

[21] Grosso J, Ocampo-Martinez C, Puig V, Joseph B. Chance-constrained model predictive control for drinking water networks. Journal of Process Control 2014; 24(5):504-516.

[22] Raso L, Schwanenberg D, van de Giesen N, van Overloop P. Short-term optimal operation of water systems using ensemble forecasts. Advances in Water Resources 2014; 71:200 - 208.

[23] Maestre JM, Raso L, Van Overloop PJ, De Schutter B. Distributed tree-based model predictive control on an open water system. American Control Conference (ACC), Montréal, Canada, 2012; 1985-1990.

[24] Lucia S, Subramanian S, Engell S. Non-conservative robust nonlinear model predictive control via scenario decomposition. Proceedings of IEEE Multi-Conference on Systems and Control (MSC), Hyderabad, India, 2013; 586-591.

[25] Grosso JM, Maestre JM, Ocampo-Martinez C, Puig V. On the assessment of tree-based and chanceconstrained predictive control approaches applied to drinking water networks. Proceedings of 19th IFAC World Congress, Cape Town, South Africa, 2013.

[26] Box GEP, Jenkins GM, Reisnel GC. Time Series Analysis, Forecasting and Control. Third edn., PrenticeHall International, Inc.: New Jersey, U.S.A, 1994.

[27] Raso L, Giesen N, Stive P, Schwanenberg D, Overloop P. Tree structure generation from ensemble forecasts for real time control. Hydrological Processes 2013; 27(1):75-82.

[28] Schildbach G, Fagiano L, Frei C, Morari M. The scenario approach for stochastic model predictive control with bounds on closed-loop constraint violations. Automatica 2014; 50(12):3009-3018.

[29] Heitsch H, Römisch W. Scenario tree modeling for multistage stochastic programs. Mathematical Programming 2009; 118(2):371-406. 
[30] Lucia S, Finkler T, Basak D, Engell S. A new robust NMPC scheme and its application to a semi-batch reactor example. In Proc. of the International Symposium on Advanced Control of Chemical Processes, Singapore 2012; :69-74.

[31] Raso L, Schwanenberg D, van der Giesen N, van Overloop PJ. Tree-scenario based model predictive control. Proceedings of EGU General Assembly Conference Abstracts, vol. 12, 2010; 3178.

[32] Walker WE, Haasnoot M, Kwakkel JH. Adapt or perish: a review of planning approaches for adaptation under deep uncertainty. Sustainability 2013; 5(3):955-979.

[33] Beh EH, Dandy GC, Maier HR, Paton FL. Optimal sequencing of water supply options at the regional scale incorporating alternative water supply sources and multiple objectives. Environmental Modelling E Software 2014; 53:137-153.

[34] Prékopa A. Stochastic Programming. Kluwer Academic Publishers, 1995.

[35] Nemirovski A, Shapiro A. Convex approximations of chance constrained programs. SIAM Journal on Optimization Dec 2006; 17(4):969-996.

[36] Ono M, Williams B. Iterative risk allocation: A new approach to robust model predictive control with a joint chance constraint. Proc. 47th IEEE Conference on Decision and Control (CDC), Cancún, Mexico, 2008; 3427-3432.

[37] Charnes A, Cooper WW. Deterministic equivalents for optimizing and satisficing under chance constraints. Operations Research 1963; 11(1):18-39.

[38] Kall P, Mayer J. Stochastic linear programming. No. 80 in International series in operations research \& management science, Springer: New York, NY, 2005.

[39] Escudero L. WARSYP: a robust modeling approach for water resources system planning under uncertainty. Annals of Operations Research 2000; 95(1-4):313-339.

[40] Rockafellar R, Wets RB. Scenario and policy aggregation in optimization under uncertainty. Mathematics of Operation Research 1991; 16(1):119-147.

[41] Maestre JM, Raso L, van Overloop PJ, De Schutter B. Distributed tree-based model predictive control on a drainage water system. Journal of Hydroinformatics 2013; 15(2):335-347.

[42] Maestre JM, Velarde P, Jurado I, Ocampo-Martinez C, Fernández I, Isla Tejera B, del Prado JR. An application of chance-constrained model predictive control to inventory management in hospitalary pharmacy. Proceedings of IEEE 53rd Annual Conference on Decision and Control (CDC), Los Angeles, California, U.S.A, 2014; 5901-5906.

[43] Schildbach G, Fagiano L, Morari M. Randomized solutions to convex programs with multiple chance constraints. SIAM Journal on Optimization Dec 2013; 23(4):2479-2501.

[44] van Overloop PJ. Model predictive control on open water systems. PhD Thesis, Delft University of Technology 2006.

[45] Growe-Kuska N, Heitsch H, Romisch W. Scenario reduction and scenario tree construction for power management problems. Proceedings of the IEEE Bologna Power Tech Conference, 2003.

[46] Sutiene K, Makackas D, Pranevicius H. Multistage k-means clustering for scenario tree construction. Informatica 2010; 21(1):123-138. 